\title{
The atmosphere, the p-factor and the bright visible circumstellar environment of the prototype of classical Cepheids $\delta$ Cep
}

\author{
Nicolas Nardetto ${ }^{1, \star}$, Ennio Poretti ${ }^{2}$, Antoine Mérand ${ }^{3}$, Richard I. Anderson ${ }^{4}$, Andrei Fokin ${ }^{5}$, \\ Pascal Fouqué ${ }^{6}$, Alexandre Gallenne ${ }^{3,7}$, Wolfgang Gieren ${ }^{7,8}$, Dariusz Graczyk ${ }^{7,8,9}$, Pierre \\ Kervella ${ }^{10,11}$, Philippe Mathias ${ }^{12,13}$, Denis Mourard ${ }^{1}$, Hilding Neilson ${ }^{14}$, Grzegorz Pietrzynski ${ }^{9}$, \\ Bogumil Pilecki ${ }^{9}$, Monica Rainer ${ }^{2}$, and Jesper Storm ${ }^{15}$ \\ ${ }^{1}$ Laboratoire Lagrange, UMR7293, Univ. de Nice Sophia-Antipolis, CNRS, Obs.de la Côte d'Azur, France \\ 2 INAF - Osservatorio Astronomico di Brera, Via E. Bianchi 46, 23807 Merate (LC), Italy \\ ${ }^{3}$ European Southern Observatory, Alonso de Córdova 3107, Casilla 19001, Santiago 19, Chile \\ ${ }^{4}$ Department of Physics and Astronomy, The Johns Hopkins University, Baltimore, MD, 21218, USA \\ ${ }^{5}$ Institute of Astronomy of the Russian Academy of Sciences, 48 Pjatnitskaya Str., 109017, Moscow, Russia \\ ${ }^{6}$ Observatoire Midi-Pyrénées, UMR 5572, Université Paul Sabatier-Toulouse, France \\ ${ }^{7}$ Departamento de Astronomía, Universidad de Concepción, Casilla 160-C, Concepción, Chile \\ ${ }^{8}$ Millennium Institute of Astrophysics, Santiago, Chile \\ ${ }^{9}$ Nicolaus Copernicus Astronomical Center, Polish Academy of Sciences, Warszawa, Poland \\ ${ }^{10}$ LESIA (UMR 8109), Obs. de Paris, PSL, CNRS, UPMC, Univ. Paris-Diderot, Meudon, France \\ ${ }^{11}$ Unidad Mixta Internacional Franco-Chilena de Astronomía, CNRS/INSU, France (UMI 3386) and Depar- \\ tamento de Astronomía Universidad de Chile, Camino El Observatorio 1515, Las Condes, Santiago, Chile \\ ${ }^{12}$ Univ. de Toulouse, UPS-OMP, Institut de recherche en Astrophysique et Planétologie, Toulouse, France \\ ${ }^{13}$ CNRS, UMR5277, Institut de recherche en Astrophysique et Planétologie, Toulouse, France \\ ${ }^{14}$ Department of Astronomy \& Astrophysics, University of Toronto, Toronto, ON, M5S 3H4, Canada \\ ${ }^{15}$ Leibniz Institute for Astrophysics, An der Sternwarte 16, 14482, Potsdam, Germany
}

\begin{abstract}
.
Even $\simeq 16000$ cycles after its discovery by John Goodricke in $1783, \delta$ Cep, the prototype of classical Cepheids, is still studied intensively in order to better understand its atmospheric dynamical structure and its environment. Using HARPS-N spectroscopic measurements, we have measured the atmospheric velocity gradient of $\delta$ Cep for the first time and we confirm the decomposition of the projection factor, a subtle physical quantity limiting the Baade-Wesselink (BW) method of distance determination. This decomposition clarifies the physics behind the projection factor and will be useful to interpret the hundreds of p-factors that will come out from the next Gaia release. Besides, VEGA/ CHARA interferometric observations of the star revealed a bright visible circumstellar environment contributing to about $7 \%$ to the total flux. Better understanding the physics of the pulsation and the environment of Cepheids is necessary to improve the BW method of distance determination, a robust tool to reach Cepheids in the Milky Way, and beyond, in the Local Group.
\end{abstract}

\footnotetext{
${ }^{\star}$ Nicolas. Nardetto@oca.eu
} 


\section{Introduction}

Classical Cepheids are yellow giants and supergiants pulsating stars used as stellar candles in the universe up to $30 \mathrm{Mpc}$. The relation between their mean absolute magnitude and the logarithm of their pulsation period, first discovered by [1], is currently used to calibrate the Type 1a supernovae luminosity relation and the expansion rate of the universe (Hubble constant, $H_{0}$; [2]). A $3.4 \sigma$ tension in $H_{0}$ is found between the distance scale calibration (Cepheids $+\mathrm{SN}$ Ia) and the value derived from the Cosmic Microwave Background (CMB) observed by Planck ([3]). There are two possibilities: uncorrected biases affect one or both approaches, or new physics has to be considered in order to take into account this tension ([2]). With the next Gaia release (DR2, expected in April 2018), the distance of about 300 Galactic Cepheids will be derived with a precision of better than $3 \%$. These distances will be used first to constrain the Cepheid period-luminosity relation, but they will also bring strong constrains on the physics of Cepheids, through the so-called inverse Baade-Wesselink (BW) method. The BW method is used to determine the distance of Cepheids in the Milky Way and beyond, in the Magellanic Clouds, and consists in combining the angular size variations of the star with its linear size variation. The angular size variation can be determined using infrared surface-brightness relations ([4, 5]), interferometry ([6]) or even a full set of photometric and interferometric data (SPIPS approach; [7]). The linear size variation is deduced from spectroscopy. The radial velocity curve is first derived from a spectral line profile or a set of spectral line profiles (cross-correlation). The radial velocity curve is then multiplied by a projection factor, which is used to derive the true pulsation velocity curve of the star. Finally this pulsation velocity curve is time-integrated in order to derive the radius variation of the star. In this approach, the projection factor and the distance of the star are fully degenerate. Thus, if the distance is known, the p-factor can be derived. This has been done for several Cepheids already $([8,9])$, but with Gaia parallaxes, it should be possible to derive the projection factor of about 300 Cepheids with a $3 \%$ precision. In this context, the p-factor decomposition into three subconcepts proposed by [10] will be useful in order to interpret the Gaia p-factors (Sect. 2). This is even more promising since the decomposition of the p-factor has been confirmed observationally in the case of $\delta$ Cep thanks to a set of HARPS-N data of exceptional quality (Sect. 3). Besides, a second limiting aspect of the BW method, and even of the period-luminosity calibration, is the circumstellar environment of Cepheids (CSE). These CSEs were already discovered in the infrared, in particular in the case of $\delta$ Cep with a K-band flux contribution of $1.5 \%$ ([11]). Recently, we discovered a little secret of $\delta$ Cep using VEGA/CHARA interferometric observations: the star appears surrounded by a bright visible circumstellar environment (CSE), which was never observed before for any Cepheid. The implication of such visible CSE on the BW method is still under investigation (Sect. 4).

\section{The physical concepts behind the p-factor}

As already mentioned the projection factor is used to convert the radial velocity into the pulsation velocity of the star. For a Cepheid described simply by a uniform disk pulsating, its value is 1.5 (whatever the pulsation phase). But actually, the radial velocity of each surface element of the star is projected along the light of sight and weighted by the intensity distribution of the Cepheid. The limbdarkening of $\delta$ Cep reduces the p-factor significantly, and the so-called geometric projection factor ( $p_{0}$, step 1 in Figure 1) is between 1.36 to 1.39 , depending on the wavelength in the visible range. The time variation of the $\mathrm{p}$-factor, due mainly to limb-darkening variation, is neglected as it has no impact on the distance ([12]). However, a Cepheid is not simply a limb-darkened pulsating photosphere, it has also an extended atmosphere with various spectral lines (in absorption) forming at different levels from which we derive the radial velocity curve used in the BW method. Moreover, there is a velocity gradient in the atmosphere of the Cepheid, which can be measured from spectroscopic 


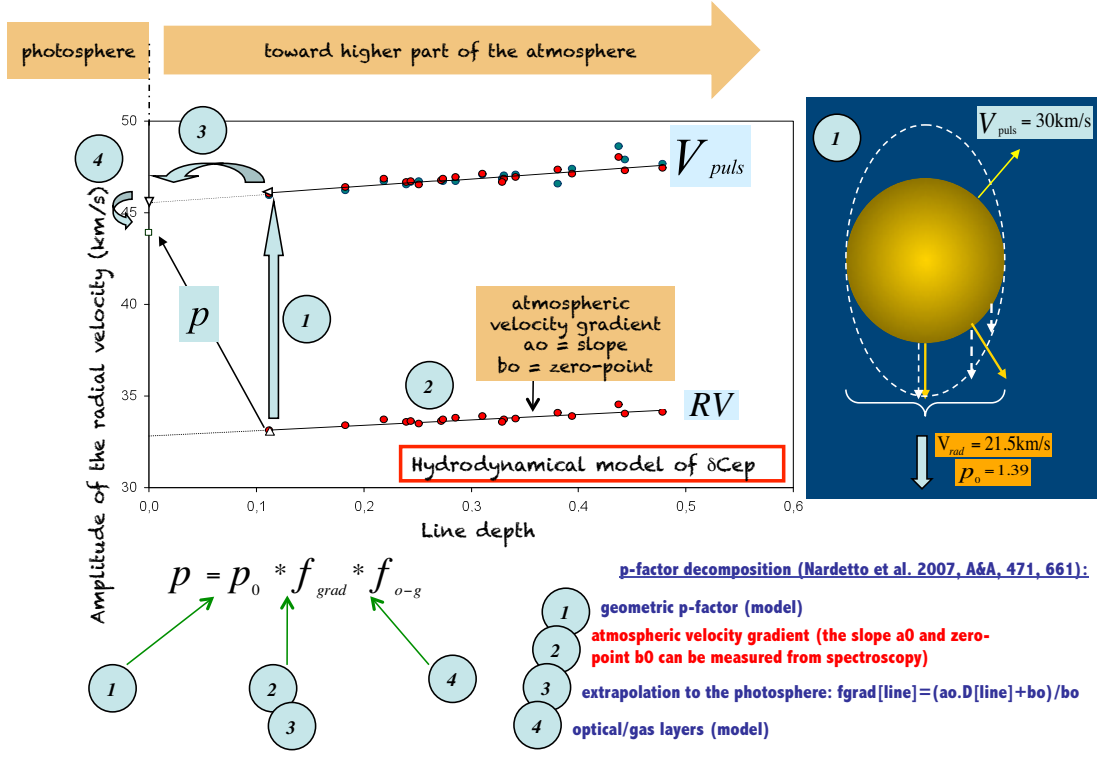

Figure 1. The p-factor decomposition is illustrated based on the hydrodynamical model of $\delta$ Cep (see [10]). The different steps are explained in Sect. 2.

observations (step 2 in Fig. 1). Then, depending on the line considered, the amplitude of the radial velocity curve will not be the same and the resulting projection factor will be different. In Figure 1 ( $f_{\text {grad }}$, step 3), we show the impact of the atmospheric velocity gradient on the p-factor for a line forming rather close to the photosphere (line depth of about 0.1 ). The higher is the line forming region in the atmosphere, the lower is the projection factor (up to $3 \%$ compared to $p_{0}$ in the case of $\delta$ Cep). The last correction on the projection factor $\left(f_{\mathrm{o}-\mathrm{g}}\right.$, step 4$)$ is more subtle. In spectroscopy, the radial velocity is actually a velocity associated with the moving gas in the line forming region, while in photometry or interferometry, we probe an optical layer corresponding to the black body continuum (i.e. the layer from which the photons escape). A correction on the projection factor of several percents (independent of the wavelength or the line considered) has to be considered. A relation between the period of Cepheids and the $\mathrm{p}$-factor has been established using this approach for a specific line ([10]) or using the cross-correlation method ([13]).

\section{The case of $\delta$ Cep revisited with HARPS-N data}

Using HARPS-N data, [14] derived for the first time the atmospheric velocity gradient of $\delta$ Cep, i.e. the amplitude of the radial velocity as a function of the line depth (step 1 in Fig. 1). In order to reproduce all observables we had to rescale consistently our hydrodynamical model of $\delta$ Cep (already published by [15]), by applying a $7.8 \%$ increase on the amplitude of the velocity and radius variation curves, which, by definition, let the projection factor unchanged. The observed and modeled atmospheric velocity gradient are plotted in Figure 2 (left). Two lines in our sample (on the lower part of the figure) do not follow the relation for unknown reasons. Beside the hydrodynamical model reproduces almost perfectly the amplitude and shape of the angular diameter curve of $\delta$ Cep obtained from FLUOR/CHARA measurements by [16] (Fig. 2, right). The distance of $\delta$ Cep is known to few percents ([17]). By considering FLUOR/CHARA interferometric measurements and this distance, it is possible to inverse the $\mathrm{BW}$ approach and derive the projection factor for all lines in our sample (see 

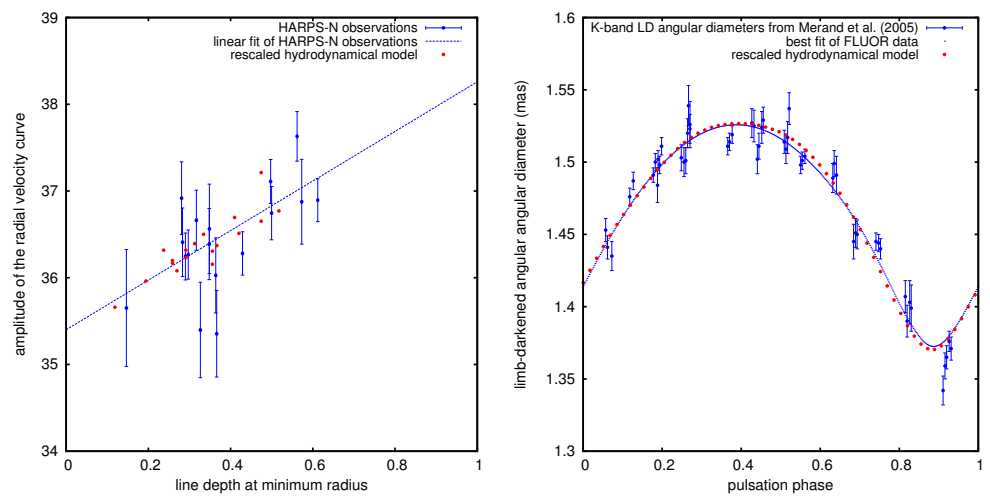

Figure 2. The rescaled hydrodynamical model of $\delta$ Cep (Fig. 1) is compared to the atmospheric velocity gradient measured by HARPS-N (left) and the angular diameter curve measured by FLUOR/ CHARA (right).
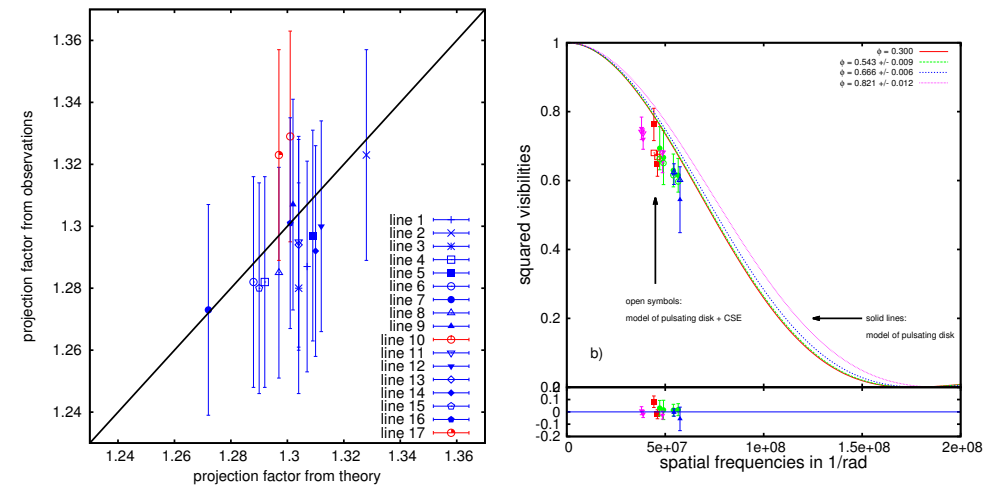

Figure 3. The observed p-factors (derived from HARPS-N, FLUOR/ CHARA and the distance to $\delta$ Cep) are compared to the theoretical ones calculated from the hydrodynamical model of $\delta$ Cep (left). An visible circumstellar environment is detected by the VEGA/CHARA interferometric measurements (right).

Table 3 in [14]). The comparison between observed and theoretical projection factors (derived from the hydrodynamical code) are plotted in Figure 3 (left). The agreement of the hydrodynamical model with the atmospheric velocity gradient, the angular diameter curve and the individual projection factors associated to specific lines, supports the decomposition of the projection factor as described in Section 2.

\section{The bright visible environment of $\delta$ Cep revealed by VEGA/CHARA observations}

It is well known now that Cepheids, and in particular $\delta$ Cep, have infrared circumstellar environments contributing to few percents of the total flux ([11]). Characterizing the CSEs of Cepheids, and in particular their radial brightness profile in several spectral bands, is of high importance in order to unbias the period-luminosity relation. Recently, we observe $\delta$ Cep with the VEGA/CHARA instrument ([18]) and we discovered an unexpected visible CSE contributing to $7 \pm 1 \%$ to the total flux, with a size of $8.9 \pm 3.0$ mas (but a visible background filling the field of view of the interferometer is not excluded; see [19]). Figure 3 (right) shows the visibility measurements associated to the shortest baseline of the CHARA interferometer (S1S2). In order to reproduce the squared visibility measurements, one needs to consider the CSE around the pulsating limb-darkened disk of the star, otherwise the model is several $\sigma$ above the measurements (whatever the observed pulsation phase considered). Furthermore, [20] found the signature of a companion in recent spectroscopic data of $\delta \mathrm{Cep}$. Its expected flux contribution is supposed to be lower than $1 \%$ in the visible, clearly not detectable by VEGA/CHARA. Interestingly, $\delta \mathrm{Cep}$ is also found to be a soft X-ray source ([21]). 
An improved BW method or SPIPS approach (with in particular well calibrated p-factors and CSEs) will provide a robust tool to derive the distance of individual Cepheids up to few Mpc in the Local Group. The problem of the metallicity dependence of distance indicators in the Local Group will be then revisited in the framework of the Araucaria Project ([22]). After a long history, the BW projection factor remains a key quantity in the calibration of the cosmic distance scale, and one century after the discovery of the period-luminosity relation, Cepheid pulsation and environment is still a distinct challenge.

Acknowledgments: The observations leading to these results have received funding from the European Commission's Seventh Framework Programme (FP7/2013-2016) under grant agreement number 312430 (OPTICON). The authors thank the CHARA Array, which is funded by the National Science Foundation through NSF grants AST-0606958 and AST-0908253 and by Georgia State University through the College of Arts and Sciences, as well as the W. M. Keck Foundation. WG gratefully acknowledges financial support for this work from the BASAL Centro de Astrofisica y Tecnologias Afines (CATA) PFB-06/2007, and from the Millenium Institute of Astrophysics (MAS) of the Iniciativa Cientifica Milenio del Ministerio de Economia, Fomento y Turismo de Chile, project IC120009. We acknowledge financial support for this work from ECOS-CONICYT grant C13U01. Support from the Polish National Science Center grant MAESTRO 2012/06/A/ST9/00269 is also acknowledged. EP and MR acknowledge financial support from PRIN INAF-2014. NN, PK, AG, and WG acknowledge the support of the French-Chilean exchange program ECOS-Sud/CONICYT (C13U01). The authors acknowledge the support of the French Agence Nationale de la Recherche (ANR), under grant ANR-15-CE310012- 01 (project UnlockCepheids) and the financial support from "Programme National de Physique Stellaire" (PNPS) of CNRS/INSU, France.

\section{References}

[1] Leavitt, H. S., \& Pickering, E. C., Harvard College Observatory Circular, 173, 3 (1912)

[2] Riess, A. G., Macri, L. M. , Hoffmann, S. L., et al., ApJ, 826, 56, (2016)

[3] Planck Collaboration, Ade, P. A. R., Aghanim, N., Arnaud, M., et al., A\&A, 594, 13 (2016)

[4] Storm, J., Gieren, W., Fouqué, P., et al., A\&A, 534, 94, (2011)

[5] Storm, J., Gieren, W., Fouqué, P., et al., ApJ, 534, 95, (2011)

[6] Kervella, P., Nardetto N., Bersier D., et al., A\&A, 416, 953, (2004)

[7] Mérand, A., Kervella, P., Breitfelder, J., et al., A\&A, 584, A80 (2015)

[8] Breitfelder, J., Mérand, A., Kervella, P., et al., A\&A, 587, 117 (2016)

[9] Kervella, P., Trahin, B., Bond, H. E., et al., A\&A, 600, A127 (2017)

[10] Nardetto, N., Mourard, D., Mathias, P., Fokin, A., \& Gillet, D., A\&A, 471, 661 (2007)

[11] Mérand, A., Kervella, P., Coudé du Foresto, V., et al., A\&A, 453, 162, (2006)

[12] Nardetto, N., Fokin, A., Mourard, D., \& Mathias, P., A\&A, 454, 332, (2006)

[13] Nardetto, N., Gieren, W., Kervella, P., et al., A\&A, 502, 956, (2009)

[14] Nardetto, N., Poretti, E., Rainer, M., et al., A\&A, 597, 73, (2017)

[15] Nardetto, N., Fokin, A., Mourard, D., Mathias, P., et al., A\&A, 428, 137, (2004)

[16] Mérand, A., Kervella, P., Coudé du Foresto, V., et al., A\&A, 438, 9, (2005)

[17] Majaess, D., Turner, D., \& Gieren, W., ApJ, 747, 145, (2012)

[18] Mourard, D., Clausse, J. M., Marcotto, A., et al., A\&A, 508, 1083, (2009)

[19] Nardetto, N., Mérand, A., Mourard, D., et al., A\&A, 593, 45, (2016)

[20] Anderson, R. I., Sahlmann, J., Holl, B., et al., ApJ, 804, 144 (2015)

[21] Engle, S. G., Guinan, E. F., Harper, G. M., et al., ApJ, 838, 67 (2017)

[22] Gieren, W., Pietrzynski, G., Bresolin, F., et al., The Messenger, 121, 28, (2005) 